\title{
XL. Narrative of experiments made with the seconds pendulum, principally in order to determine the hitherto unassigned amount of the influence of certain minute forces on its rate of motion
}

\section{Mr. James Scrymgeour}

To cite this article: Mr. James Scrymgeour (1833) XL. Narrative of experiments made with the seconds pendulum, principally in order to determine the hitherto unassigned amount of the influence of certain minute forces on its rate of motion, Philosophical Magazine Series 3, 2:10, 244-251, DOI: $10.1080 / 14786443308648027$

To link to this article: http://dx.doi.org/10.1080/14786443308648027

册 Published online: 01 Jun 2009.

Submit your article to this journal 준

Џll Article views: 2 


\section{Mr. Scrymgeour's Experiments to determine the Influence}

the fact of the irruption of the sea by destroying the ancient dykes, nor with regard to the period which they assign as the date of that event*.

XL. Narrative of Experiments made with the Seconds Pendulum, principally in order to determine the hitherto unassigned Amount of the Infuence of certain minute Forces on its Rate of Motion. By Mr. James Scrymgeourt.

THE author's object in making the experiments detailed in the following narrative, was so much ramified in the course of his researches, that it will be best understood after their perusal.

Suffice it at present to say, that he had in view to ascertain the influence of the maintaining power, or escapement on the time of the vibrations of the pendulum in which no recoil is produced; and likewise the effect of the resistance of the air upon the vibrations, either when the pendulum is attached to a clock, or when it is in a detached state.

The results of these experimental researches will be found applicable to all the contrivances or escapements for giving the impulse to the pendulum without recoil. As recoil was considered to introduce sources of irregularity, it was deemed unnecessary to inquire into its effects, as they would be indefinite and would vary with the quantity of recoil ; besides, such experiments would be applicable to the clock employed only.

In pursuing this inquiry, my first object was to make the suspending spring of two of my clocks (which had both dead escapements) such as to cause the long and short vibrations to be performed in equal times. For this purpose, I shortened the acting part of the suspending spring of one of them, which, for the sake of distinction, I shall call my shop time-piece. This clock has a common lenticular bob with a wooden rod; it had been going for five or six years previously, with the suspending spring made in the usual way, of a piece of middlesized watch main-spring about $\frac{3}{4}$ of an inch in length. The bob is only about 4 pounds in weight, with a small brass ball below it, nearly $\frac{1}{2}$ of a pound in weight.

The action of the suspending spring was shortened by fixing a piece of steel on each side of the spring, and joining it to the rod, so as to reduce the acting part of the spring to

* See the Cambro-Briton, for June 1820, vol. i. p. 361 ; Edward Williams's Lyric Poems, vol. i. p. 78. note; Davies's Mythology and Rites of the Druids, p. 240, \&c.; Meyrick's Hist. and Antiquities of the County of Cardigan, p. 50-80.

+ Communicated by the Author. 


\section{of minute Forces on the Rate of the Seconds Pendulum. 245}

about $\frac{1}{8}$ of un inch in length. I was so fortunate as to obtain my object on the first trial; for when the vibrations were made to vary from $1^{\circ} \cdot 5$ to $2^{\circ} \cdot 5$, on each side of the point of rest, they were equal to within less than half a second in 24 hours.

I next adjusted the suspending spring of the other clock, which had a mercurial pendulum, and a suspending spring nearly $\frac{1}{2}$ of an inch in breadth, and $T^{\frac{1}{2}} \frac{0}{0}$ of an inch in thickness. A moveable clamp-piece was made to fasten near the top of the pendulum rod, so as to be shifted up and down at pleasure, and to clasp the suspending spring. By various trials I found, that for this strength of spring and weight of pendulum, the acting part required to be about $\frac{1}{2} \sigma$ of an inch in length.

After both clocks had been going for some time, I was surprised to find that each of them experienced a losing rate, but particularly the shop time-piece with the light bob. Since that period, which is about four years, I have had ample opportunities of satisfying myself that this loss of rate originated in the weakening of the spring.

The clamping or fastening of the spring in the manner described, without any shortening of the absolute length of the pendulum, occasioned the clock having the light pendulum to gain about 3 minutes in 24 hours. I therefore lowered the pendulum bob, and brought it to time. About a month afterwards, I found it losing on its rate, and screwed up the pendulum bob. It is now (at the distance of nearly four years) screwed up to about half of the distance it was lowered at first; a circumstance which shows how much the spring has weakened since that time. The clock frequently maintained a uniform rate for three or four weeks successively; after which it began to lose on its rate, particularly when any sudden change of temperature occurred.

Pendulums with thin suspending springs (which most of the best clocks have) will not be subject to this variation, after having gone for some time, at least as far as my experience enables me to judge.

The clock just mentioned was lately cleaned. Before cleaning, it lost about a second in 24 hours, and the extent of its vibrations was reduced to $2^{\circ}$; after cleaning, the extent of its vibrations was $2^{\circ} \cdot 5$, its original extent when clean; it then gained 5 seconds in 24 hours, making a difference of 6 seconds all together. The cause of this difference was, less friction on the pallets.

The impulse being given in the ascent occasioned all the friction that affected the time to be in the descent, which con- 
sequently retarded the motion and caused the time to be slower. The friction beyond the escaping point being equal in ascent and descent, occasions no difference in time.

I next put a lighter maintaining power on the clock, which reduced the extent of vibration to $2^{\circ}$, its extent before it was cleaned; but it still gained 5 seconds in 24 hours. This result shows that the spring was such as to cause the vibrations to be equal in time, and that the loss in its rate before cleaning was occasioned by friction.

I was now desirous of ascertaining the rate of the pendulum of this clock in a detached state. For this purpose, I suspended a detached pendulum, with an adjusted suspending spring, close in front of the clock, and adjusted it so as to vibrate with the clock pendulum. I then detached the pallets from the crowri-wheel. The collet to which the pallets were riveted being fixed to the arbor by a screw, they could thus be put out of action, clear of the wheel, and fixed again upon the arbor. In this construction, the pallets, with the arbor and back-fork, form part of the pendulum.

The pendulum was put in motion by the hand, a little beyond its extent of vibration, so that the mean extent in the detached state should be the same as when attached to the clock. The experiment showed that it gained upon the detached pendulum. I then adjusted it to the detached pendulum, and put it in action with the work as before. It now lost about $2 \frac{1}{2}$ seconds in 24 hours upon its former rate. This experiment, therefore, showed the influence of the escapement upon the time of the vibrations, which was occasioned, conformably to what has been already stated, by the remain-ing friction upon the pallets in the descent, in consequence of the impulse being given in the ascent.

It is proper to mention here, that the bob of this clock traversed close to the back of the case; and the resistance thus occasioned, accounts for part of the loss of $2 \frac{1}{2}$ seconds, as will be shown hereafter.

The same experiment was next tried with the clock having the mercurial pendulum, of about $13 \frac{1}{2}$ pounds in weight, and furnished with the strong adjusted suspending spring. The result showed a loss amounting to within 2 or 3 tenths of a second of the loss of rate exhibited in the former experiment.

In order to give the impulse in the descent instead of the ascent, the following means were adopted. The flanches of the pallets were hollowed by forming them thus: $\square$ with a small cylindrical lap of about $\frac{1}{8}$ of an inch in diameter. These pallets were made separate, and being jointed upon their axes of motion, the distance between them was regulated by 
a tangent-screw, so that they could be adjusted to any depth in the teeth of the wheel. This shape afforded the means of giving about three fourths of the impulse in the descent. Experiment showed that the clock now gained about 2 seconds in 24 hours. The extra friction in this case was in the ascent.

It is known that if a pendulum have at the point of rest impulse sufficient to reach $2^{\circ}$, with a certain quantity of friction, it will require a greater impulse with a greater quantity of friction. The greater impulse will of course cause the first increments of motion to be quicker in the latter case than in the former; but this incidental quickness will have gradually decreased, and vanished when the pendulum has arrived at the extent of $2^{\circ}$; and consequently, the entire vibration will be quicker. Pendulums having strong adjusted suspending springs require a maintaining power in weight about double of that which they would require with a thin spring, or on a knife-edge, in order to make them vibrate to the extent of $2^{\circ}$.

The change of rate occasioned by the use of strong suspending springs, led to the consideration of some other practical means for obtaining isochronism, cycloidal cheeks being admitted to be objectionable in practice. It occurred to me that by placing a cylinder, or a portion of it, at the point of suspension of a pendulum, and making it roll on a plane, the point of oscillation would move in a cycloid, at least as far as the extent of the vibrations of the pendulum required. For this purpose, I constructed an appropriate apparatus. I described the central portion of a cycloid on a sheet of brass, and fixed it on a wall, with a horizontal plane on the same, at a proper distance above for the length of the seconds pendulum. After several trials with cylinders of different sizes, it appeared that one of about 2 inches in diameter caused the point of oscillation to traverse in the curve, at least as far as could be sensibly determined.

These trials led to the construction of a cylinder the size of which was a very near approximation to the proper magnitude. This cylinder was then fixed to a convertible pendulum, which I had previously constructed; but in the present case it was used as a common pendulum. The result of more careful and repeated experiments showed that a cylinder of 1.8 inch in diameter caused the seconds pendulum to perform its vibrations in times more nearly equable than could be obtained by cylinders of any other size.

Thinking, however, that the property of isochronism might possibly depend on some minute size or proportion, I first shortened the pendulum, making use of the cylinder which gave the nearest approximation. I then gradually and suc- 
cessively lengthened it, even beyond its proper length, and tried the vibrations at every small interval; but the experiments failed to give a nearer approximation to isochronisn than in the former case. In short, the vibrations thus obtained did not appear to be more isochronous than those which were obtained by employing a knife-edge.

Being disappointed in not finding the vibrations equal in time, I began to doubt the property of the cycloid in rendering the vibrations of the pendulum isochronous. For my own satisfaction on this point, I therefore instituted the following experiments. I described a circle with beam compasses (the distance of the points of which was double the diameter of the circle with which the cycloid had been described), so close by the central portion of the cycloid on the same sheet of brass, as to be within $\frac{1}{2} 0$ of an inch of that curve. To an extent of about $9^{\circ}$ or $10^{\circ}$ on each side of the middle of the curve, there was no perceptible difference between it and the circumference of the circle to the same extent.

My next step was to construct metallic cycloidal cheeks of the proper size for the seconds pendulum, and to fix them in a vertical position, so that their extreme points were at the distance of the base of the curve from each other; while the middle portions of the curve inverted served as cheeks for the vibrations of the pendulum, in the usual way prescribed in philosophical treatises on the subject. A ball was then suspended between the cheeks, by means of a piece of small gold wire flattened to about $3^{\frac{1}{0} \delta}$ of an inch in thickness. As soon as the ball was made to vibrate, it was obvious that the cheeks would have no effect in equalizing the vibrations, even when the extent of vibration was $9^{\circ}$ or $10^{\circ}$ beyond the point of rest.

Subsequently, it occurred to me to try the experiment by employing the extreme portions of the curve as cheeks, and fixing them for this purpose in the manner above mentioned. The cycloidal cheeks formed in this manner were found to have a considerable effect in equalizing the times of the vibrations; for the short vibrations of $1^{0.5}$ were not above 8 or 10 seconds faster in 24 hours than the longer vibrations of $4^{\circ}$ or $5^{\circ}$; and had all been as perfect as theory requires, the vibrations might have been isochronous.

In ascertaining the proportions of the times of the long and short vibrations, the detached pendulum was always adjusted to the clock pendulum, so as to make them vibrate alike. The fixture for the detached pendulum was a little in front of the clock; and the two pendulums were observed by viewing them from such a point that they were both seen in a line when at the extent of their vibrations; the experiment being always 
commenced by putting them in motion so as to vibrate exactly with one another. This method was considered not only more simple than that of coincidences, but fully as accurate. It is also more suitable for an experimenter who cannot conveniently have an assistant at all times; nor indeed would the method of coincidences have answered so well for the various experiments which I had in view.

Though the preceding experiments occupied upwards of 12 months, during all my spare hours from business, either in making the necessary apparatus or the experiments themselves, yet considerable practical advantage was thereby obtained.

The object of the following experiments was to ascertain the difference in time between the vibrations of a pendulum in air and in vacuo, \&c. For the purpose of making experiments in vacuo, or in an exhausted vessel, the following apparatus was constructed.-An iron vessel was made, having circular apertures about 6 inches in diameter, near the bottom, at a proper distance for observing the extremity of the pendulum rod. These apertures were glazed with strong plateglass, so as to be capable of bearing the great pressure consequent on a high degree of rarefaction. The vessel was placed close in front of the clock-case, which had sides made to withdraw, so as to admit more readily of the adjustment of the pendulum, \&c. There was likewise in the vessel a contrivance for putting the pendulum in motion at any degree of rarefaction or exhaustion.

In his experiments on the pendulum, Captain Kater appears to consider that the buoyancy of the air causes the vibrations of a pendulum to be slower. "Thus," says he, " the specific gravity of water compared with that of air, may be known for the temperature and altitude of the barometer at the time of observation; and multiplying this by the specific gravity of the pendulum, the ratio of the weight of the pendulum compared with that of air will be obtained. This ratio will express the diminution of the force of gravity arising from the buoyancy of the atmosphere: and in order that the number of vibrations may be the same in vacuo as in air, the length of the penduluin must be increased in the proportion of this ratio to 1 , the lengths of pendulums vibrating in the same time, varying directly as the force of gravity."

Buoyancy is here considered equivalent to a diminution of gravity, and proportional to the deduction of the weight of the pendulum's bulk of air from its own weight; consequently, it should appear as if a light pendulum would vibrate more slowly than a heavy one. Not being of this opinion, I made the following experiment to put it to the test. I mounted a

Third. Series. Vol. 2. No. 10. April $1833 . \quad 2 \mathrm{~K}$ 
light pendulum with a wooden bob, of a cylindrical form, about $4 \frac{1}{2}$ inches long and 2 inches in diameter; this bob was 10 ounces in weight, and, along with a brass ball and the pendudulum rod, weighed about 15 ounces. A piece of thin iron, about $\frac{3}{4}$ of an inch in breadth, was fixed to the end of the pendulum rod, edgewise to the direction of motion of the pendulum. The object in making it so light was, that the effect of buoyancy might be more readily observed, and also its difference of rate in air and in vacuo.

The pendulum thus mounted was suspended in the vessel in which the vacuum or exhaustion was to be produced. $\mathbf{A}$ small trough of mercury, about 6 inches long, 1 inch deep, and 1 ineh broad, was placed at the bottom of the vessel, having its length parallel to the direction of motion of the pendulum. After frequent adjustments and exhaustions, I succeeded in making the lower edge of the piece of iron traverse slightly in the mercury, so that at the end of each vibration it was nearly free of the surface. Some dust upon this surface indicated a slight motion in the direction of the pendulum; but it almost ceased with the motion of the pendulum at its extent of vibration, and appeared to produce no effect upon the time in descending.

By such means, the pendulum was made to lose its extent of motion nearly at the same rate as a pendulum with a metallic bob does in air; the clock pendulum was adjusted to vibrate with the light pendulum. The trough with the mercury was afterwards removed, the vessel was exhausted to the same degree as before, and the same mean extent of vibration was employed; but careful experiment showed no perceptible difference in time.

In this experiment, I considered that the mercury should buoy up the pendulum as much as air; and if so, we must evidently conclude from the result, that buoyancy makes no difference upon the time of the vibrations of a pendulum. When the same pendulum used above was made to vibrate in air; it lost one vibration in 20 minutes upon the clock pendulum.

By this time I had begun to suspect the true cause why the pendulum lost time when vibrating in air, and my conclusion was as follows: A current was generated in the air by the motion of the pendulum, and in the direction of its motion; consequently, when the pendulum reached its height and its momentum was exhausted, the current thus generated now slightly suspended its motion, and retarded the first increments of its descent.

The current of air generated by the vibrations of the bob of a pendulum may be rendered distinctly visible thus : Fix a 
piece of rag at the bottom of the pendulum, set fire to the rag, and blow out the flame, still allowing the rag to smoke; then put the pendulum in motion, and the smoke will be seen passing along in the direction of the pendulum's motion, even before it can be observed that it has begun to descend.

Had Captain Kater been aware of this fact, he would have been at no loss to account for his pendulum losing its adjustment, when by the hygrometer he observed a great and sudden change in the air from moisture to dryness. This observation shows the accuracy with which his experiments were conducted; it also indicates that dry air is more dense than moist, as the current generated in the latter state offered more resistance to the return of the pendulum than in the former.

For, in his experiments, at the time when the smaller weight of the pendulum was down, the vibrations would be slower than when the greater weight was down; the smaller weight presenting a larger surface to the resisting medium, in proportion to its weight, than the greater weight, the current then generated would oppose its descent more than the current generated by the greater weight.

[To be continued.]

XLI. On the Theory of Voltaic Action. By Mr. JoHn Prideaux.

[Concluded from p. 220.]

Sect. IV. Of the Conducting Property of the Liquid.

25. THE conducting power of the liquid is a main point in voltaic phænomena; and acid liquids are understood, generally, to be the best conductors (liquid metals of course left out of the question); alkaline liquids the worst, of aqueous solutions; and alcohol, oils, and the like, as nonconductors. Thus, from whatever kind of coincidence, the conducting and electro-negative properties seem to bear some mutual relation.

26. Whether this conduction in the voltaic battery be from particle to particle, or, like that of caloric in liquids, connected with transference of the particles themselves, becomes the next inquiry. Of such transference we have abundant evidence; but in what degree it is essential to the conducting process, it may be difficult to ascertain by direct experiment.

27. Separate glasses, filled with acid and alkaline solutions, and connected by a siphon filled with water, separated cells, similarly filled, and divided by bladder, each having a copper plate plunged in the alkali, a zinc plate in the acid, and con$2 \mathrm{~K} 2$ 